\title{
Resultados del tratamiento quirúrgico de las metástasis hepáticas por cáncer colorrectal
}

\author{
Enrique Norero ${ }^{1}$, Nicolás Jarufe ${ }^{1}$, Jean $\mathrm{M} \mathrm{Butte}^{1}$, \\ Blanca N orero ${ }^{1}$, Ignacio Duarte ${ }^{2}$, Javiera Torres ${ }^{2}$, \\ George Pinedo ${ }^{1}$, Francisco López ${ }^{1 *}$, Juan Francisco \\ G uerra' ${ }^{1}$ Luis Ibáñez ${ }^{1}$, Álvaro Zúñiga ${ }^{1}$, Sergio G uzmán ${ }^{1}$, \\ Jorge Martínez ${ }^{1}$.

\section{O utcome of surgical treatment of liver metastasis from colorectal cancer}

Background: Surgical resection is the only treatment associated with long-term cure in patients with liver metastasis from colorectal cancer, achieving a $30 \%$ to $40 \%$ five years survival. Aim: To evaluate the results of liver resection for metastatic colorectal cancer in our centre. Patients and methods: Retrospective study. Epidemiological, perioperative and follow up data of patients undergoing liver resection for metastatic colorectal cancer between January 1990 and July 2007 were assessed. We compared the results between two periods; period 1 (1990-1997) and period 2 (1998-2007). Results: Sixty six patients aged $61 \pm 12$ years (46 males) underwent 75 resections. An anatomical excision was performed in 54 (72\%) cases, a right hepatectomy in 18, an extended right hepatectomy in 11 , a left hepatectomy in 1, and a segmentectomy in 24 . In $24(32 \%)$ patients the liver resection was simultaneous with the colorectal cancer resection. Operative time was $221 \pm 86$ min. Hospital stay was $11 \pm 5$ days. Postoperative morbidity was $35 \%$ and surgical mortality was $0 \%$. Resection margin was free of tumor in $53(80 \%)$ patients. Five years overall and hepatic disease-free survival was $38 \%$ and $23 \%$, respectively. In period 2 , more anatomical resections than in period 1 were performed ( $77 \%$ and $55 \%$, respectively, $\mathrm{p}=0.04$ ), without an increase in complications ( $35 \%$ and $34 \%$, respectively; $p=n s)$, but with a better five years survival $(45 \%$ and $21 \%$, respectively, $p=0.04$ ). Conclusions: Five years survival for excision of liver metastatic colorectal cancer in our center is similar to that reported abroad. During the second period there has been a trend toward more extensive resections which was associated with a better survival, without an increase in complications or mortality (Rev Méd Chile 2009; 137: 487-96). (Key words: Colorectal neoplasms; Neoplasm metastasis; Survival)

Recibido el 22 de julio, 2008. Aceptado el 24 de diciembre, 2008.

${ }^{1}$ Departamento de Cirugía Digestiva, División de Cirugía. ${ }^{2}$ Departamento de Anatomía Patológica, Facultad de Medicina, Pontificia Universidad Católica de Chile. Santiago de Chile. *Afiliación actual: Clínica Las Condes.

Correspondencia a: Dr. Nicolás Jarufe. Departamento de Cirugía Digestiva, Facultad de Medicina, Pontificia Universidad Católica de Chile. Marcoleta 367, Casilla 114-D, Santiago.

Fono: 3543870. Fax: 6329620. E mail: njarufe@med.puc.cl 
$\mathrm{D}$ urante las últimas décadas se han producido considerables avances en los resultados de la cirugía hepatobiliar ${ }^{1}$. En este contexto, se ha incrementado significativamente la cirugía resectiva de las metástasis hepáticas de origen colorrectal (MHCCR) $)^{2-7}$.

En el momento del diagnóstico de un cáncer colorrectal $15 \%$ a $20 \%$ de los pacientes presentan metástasis hepáticas y entre $25 \%$ y $40 \%$ las desarrollaran en el seguimiento ${ }^{8,9}$. Estos pacientes sin tratamiento tienen una mediana de sobrevida de 10 meses $^{9,10}$. Con quimioterapia paliativa la sobrevida puede alcanzar entre 14 y 16 meses $^{11-}$ 14. El único tratamiento que ofrece la posibilidad de curación es la resección completa de las metástasis (R0), con sobrevida a 5 años de 33\%$37 \%$ y a 10 años de $20 \%-22 \%$ en grandes centros ${ }^{4,6}$.

En nuestro país comunicaciones previas han descrito un pequeño número de enfermos que puede ser sometido a esta terapia ${ }^{10,15}$. El objetivo de este trabajo es evaluar los resultados de la resección hepática por MHCCR, describir su morbilidad y mortalidad y analizar la sobrevida alejada observada y sus factores.

\section{MATERIAL y MÉTODO}

Estudio retrospectivo a partir de una base de datos especialmente diseñada; desde el año 2003 ésta se llenó prospectivamente. Se incluyó a todos los pacientes sometidos a una resección hepática por MHCCR entre enero 1990 y julio 2007. En general, en pacientes en buenas condiciones, los criterios de resección fueron: ausencia de enfermedad diseminada extrahepática no resecable, según TAC de tórax, abdomen y pelvis, resonancia magnética y en algunos casos tomografía por emisión de positrones (PET) y una evaluación del parénquima hepático remanente que fuera suficiente de acuerdo a imágenes (volumetría por $\mathrm{TAC})^{16}$.

Se analizaron datos del paciente, del tumor colorrectal primario, características de las metástasis hepáticas, tiempo entre la cirugía colorrectal y la cirugía hepática, características de las resecciones del hígado, morbilidad y mortalidad, duración de la hospitalización y de estadía en la Unidad de Cuidado Intensivo (UCI), estado del margen de resección hepática, tratamiento adyuvante realizado y sobrevida alejada.

El seguimiento se realizó mediante los registros clínicos, información del médico tratante y con los certificados de defunción del Registro Civil. Se determinó la presencia de los factores pronósticos del Memorial Sloan-Kettering Cancer Center $^{6}$ (FPMSK) (Tabla 1). Se comparó 2 períodos: período 1 (1990-1997) y período 2 (19982007) según el número y tipo de resección hepática, complicaciones, factores pronósticos, quimioterapia adyuvante, resultado de la cirugía y sobrevida alejada. Se escogió el año 1998 como corte, debido a que a partir de esta fecha hubo un cambio en el manejo de esta patología. Se favoreció realizar resecciones anatómicas y hepatectomías extendidas.

Definiciones. Se utilizó la segmentación hepática funcional de Couinaud ${ }^{17}$ y la clasificación de resecciones hepáticas del Consenso de Brisbane ${ }^{18}$ (Figura 1).

Se consideró: Resección anatómica a toda resección hepática que incluyó 1 ó más segmentos hepáticos de Couinaud. Resección local o no anatómica a la resección de parénquima hepático de menos de un segmento hepático o que no siguió la anatomía segmentaria de Couinaud. Morbilidad postoperatoria: cualquier complicación ocurrida durante la hospitalización y hasta 1 mes posterior a la intervención quirúrgica ${ }^{19}$. Insuficiencia hepática: niveles de bilirrubina elevados por sobre $6 \mathrm{mg} / \mathrm{dl}$, tiempo de protrombina $>24 \mathrm{~s}$ que hace necesario el aporte de plasma fresco congelado y elevación de niveles de transaminasas asociado a encefalopatía ${ }^{19}$. Quimioterapia de $2^{\text {a }}$ línea: cualquier esquema que incluyera irinotecan, oxaliplatino, o anticuerpos (cetuximab o bevacizumab). Se consideró recurrencia hepática a la reaparición de enfermedad neoplásica posterior a un mes desde la hepatectomía.

Estadística. Se utilizó programas estadísticos Statview 5.0.1 y Minitab 15. En la comparación de datos se aplicaron las pruebas de chi-cuadrado, $t$ Student y Fisher según correspondiera. Se calculó la sobrevida actuarial y la sobrevida libre de enfermedad hepática según Kaplan-Meier y se compararon con el test de log-rank. Se consideró significativo un valor $\mathrm{p} \leq 0,05$. Los datos se 
Tabla 1. C riterios pronósticos del Memorial Sloan-Kettering $C$ ancer $C$ enter presentes en los pacientes sometidos a resecciones hepáticas por M H C CR

\begin{tabular}{|c|c|c|c|}
\hline \multicolumn{3}{|l|}{ Criterios } & $\%$ \\
\hline \multicolumn{3}{|c|}{ Linfonodos del tumor primario $(+)$} & $59 \%$ \\
\hline \multicolumn{3}{|c|}{ Tiempo entre tumor primario y metástasis hepática $<12$ meses } & $58 \%$ \\
\hline \multicolumn{3}{|c|}{ Número de nódulos hepáticos >1 } & $50 \%$ \\
\hline \multicolumn{3}{|c|}{ Tamaño del nódulo mayor $\geq 5 \mathrm{cms}$} & $36 \%$ \\
\hline \multicolumn{3}{|c|}{ Antigeno carcinoembrionario $\geq 200$ (ng/ml) } & $11 \%$ \\
\hline Puntaje & Pacientes & Sobrevida global & Sobrevida libre enfermedad \\
\hline 1 & 18 & 55 meses $(43 \%)$ & 41 meses (38\%) \\
\hline 2 & 20 & 44 meses $(38 \%)$ & 34 meses $(27 \%)^{\dagger}$ \\
\hline 3 & 17 & 38 meses $(33 \%)^{*}$ & 15 meses $(12 \%)^{\dagger}$ \\
\hline 4 & 8 & 26 meses $(18 \%)^{*}$ & 5 meses $(0 \%)^{\dagger}$ \\
\hline
\end{tabular}

Sobrevida expresada en mediana (\% sobrevida 5 años).

MHCC: Metástasis hepáticas de origen colorrectal.

Hay 4 pacientes con puntaje 0 , todos vivos con seguimiento de 30, 85, 34 y 50 meses.

No hay pacientes con puntaje 5 .

*Puntaje 4 vs $0-3, p=0,048$; Puntaje $3-4$ vs $0-2, p=0,024$.

†Puntaje 4 vs $0-3, p=0,002$; Puntaje $3-4$ vs $0-2, p=0,001$; Puntaje 2-4 vs $0-1, p=0,001$.

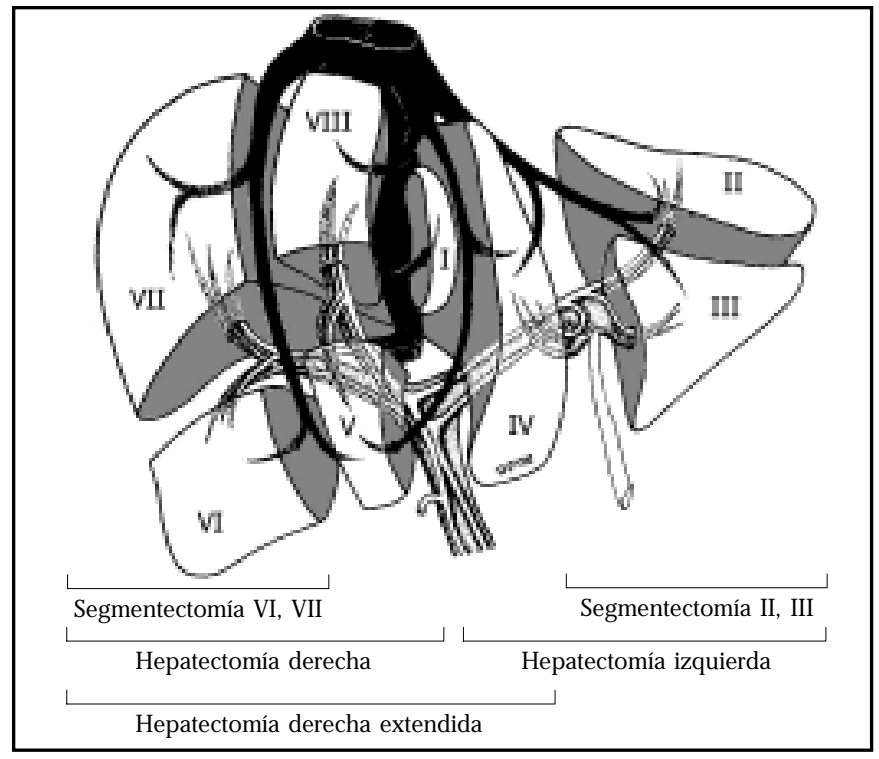

Figura 1. Anatomía segmentaria del hígado según Couinaud y tipos de resecciones hepáticas.

expresaron en medianas (rango) o promedios \pm desviación estándar y la sobrevida y seguimiento, como mediana en meses y porcentaje de sobrevida a 5 años.
Resultados

Durante el período del estudio se realizaron 75 resecciones por MHCCR en 66 pacientes (hom- 
bres: 46; edad: $61 \pm 12$ años, rango 23-92). El tumor primario se ubicó en el colon en 47 (71\%) casos y en el recto en 19 pacientes (29\%); el cáncer presentó ganglios positivos en 59\%. La etapificación del cáncer primario en el momento de su presentación inicial fue: etapa I en 4 (6\%), etapa II en 9 (14\%), etapa III en 21 (32\%) y etapa IV en 28 (42\%) y no estaba disponible en 4 pacientes (6\%).

En 57 (86\%) pacientes se realizó 1 resección hepática y en $8(12 \%)$ se practicó una segunda hepatectomía, debido a una recurrencia de MHCCR. El tiempo entre ambas resecciones hepáticas fue de 20 meses (rango: 7-38).

En $45(60 \%)$ casos se efectuó una resección anatómica, en 21 (28\%) una resección local y en 9 (12\%) ocasiones, se realizó una resección anatómica acompañada de una resección local. En el grupo de resecciones anatómicas se extiparon 3,2 $\pm 1,4$ segmentos por intervención y se realizaron 11 (15\%) resecciones extendidas (Tabla 2). Entre los 28 pacien- tes con MHCCR en el momento de la presentación inicial (etapa IV), en 24 (32\%), se realizó la resección hepática en forma simultánea con la cirugía colomectal, mientras que en los 4 restantes la resección hepática fue diferida. En un paciente se realizó una hepatectomía en 2 tiempos como estrategia planificada con intención curativa en un intervalo de 4 meses.

El tiempo operatorio fue de $221 \pm 86 \mathrm{~min}$ y el sangrado de $835 \pm 825 \mathrm{ml}$. Se realizaron transfusiones de glóbulos rojos en el intraoperatorio en $27 \%$ de las intervenciones; en promedio 2,4 $\pm 2,0$ unidades por cirugía. Se utilizó isquemia pedicular controlada ${ }^{20,21}$ (maniobra de Pringle) en 33\% de las resecciones, con una duración de $31 \pm 15$ min (rango: 5-60).

La estadía hospitalaria fue de $11 \pm 5$ días y 22 pacientes (29\%) necesitaron estadía en UCI de 2 días (1-5).

Se observó morbilidad en $35 \%$ de la serie (Tabla 3). Cuatro pacientes presentaron insuficiencia hepática; ésta fue autolimitada y respondió

Tabla 2. Resecciones hepáticas por metástasis colorrectal

\begin{tabular}{|lrr|}
\hline Resección hepática & $\mathbf{n}$ & $\%$ \\
\hline Hepatectomía derecha & 18 & 24 \\
Hepatectomía derecha extendida & 11 & 15 \\
Segmentectomía 2,3 & 9 & 12 \\
Segmentectomía 6,7 & 5 & 7 \\
Segmentectomía 5 & 2 & 3 \\
Segmentectomía 5,6 & 2 & 3 \\
Hepatectomía izquierda & 1 & 1 \\
Segmentectomías (otras) & 6 & 8 \\
No anatómica (resección local) & 21 & 28 \\
Total & 75 & 100 \\
\hline
\end{tabular}

Tabla 3. Complicaciones de las resecciones hepáticas

\begin{tabular}{|lcc|}
\hline Complicación & $\mathbf{n}$ & $\%$ \\
\hline Colección intrabdominal* & 7 & 9 \\
Fístula biliar & 6 & 8 \\
Insuficiencia hepática & 4 & 5 \\
Infección herida & 3 & 4 \\
Hemoperitoneo* & 3 & 3 \\
Neumonía & 2 & 3 \\
Otras & 11 & 14 \\
Total casos con complicaciones & 26 & 35 \\
\hline
\end{tabular}

*Reoperaciones: Una colección intrabdominal y 3 hemoperitoneos. 
con manejo médico habitual. No hubo mortalidad perioperatoria. No se encontró una diferencia significativa en morbilidad entre cirugía simultánea, colorrectal-hepática y cirugía hepática diferida en un segundo tiempo ( $21 \%$ vs $41 \%$; $p=n s$ ). Tampoco se observó diferencia en morbilidad entre la segunda hepatectomía y la primera resección ( $33 \%$ vs $50 \%$; $\mathrm{p}=\mathrm{ns})$.

El análisis de las piezas operatorias se muestra en la Tabla 4. Se obtuvo parénquima hepático libre de tumor (R0) en 53 (80\%) pacientes, en 6 (9\%) pacientes el borde microscópico fue positivo para tumor (R1) y en 7 (11\%) quedaron lesiones macroscópicas (R2).

El seguimiento fue de 100\%. La mediana de seguimiento fue de 24 meses (4-208) y el promedio de 36 meses. Treinta y siete (56\%) pacientes se encontraban vivos a los 29 meses. La sobrevida global de la serie fue de 41 meses (38\% a 5 años) (Figura 2). En los pacientes con una cirugía curativa (R0), la sobrevida fue de 50 meses ( $43 \%$ a 5 años) ( $p=0,003)$. En los pacientes con borde microscópico positivo (R1) ésta fue de 21 meses (33\% a 5 años), en cambio en los pacientes con resecciones R2 no se obtuvo sobrevida a 5 años, con una mediana de 11 meses (Figura 3).
Veintiséis pacientes (39\%) estaban vivos y sin recurrencia a los 26 meses de seguimiento (4-208). La sobrevida libre de enfermedad fue de 23 meses (23\% a 5 años) (Figura 2). Veintidós (33\%) pacientes presentaron recurrencia hepática de su enfermedad a los 15 meses (2-41). Ocho (36\%) de estos pacientes fueron sometidos a una segunda hepatectomía con márgenes de resección negativos (R0). Siete de ellos, están vivos con un seguimiento de 35 meses (25-91). El paciente restante, falleció a los 50 meses. Once pacientes fallecen con recurrencia extrahepática en diferentes sitios, pero sin evidencia de recidiva en el hígado.

La Tabla 1 resume el número y porcentaje de FPMSK y su relación con la sobrevida global y sobrevida libre de enfermedad de los pacientes de la serie. El número promedio de FPMSK fue 2,1 \pm 1. Veinticuatro (36\%) pacientes presentaron 30 más factores pronósticos. Los pacientes con un puntaje de 0 a 2 tuvieron una sobrevida de 55 meses ( $45 \%$ a 5 años); en aquéllos con un puntaje de 3 o más, la sobrevida fue de 26 meses ( $26 \%$ a 5 años) ( $p=0,024)$. Cuando se analizó la recidiva hepática, se observó que los pacientes con puntaje de 0 a 2 obtuvieron una sobrevida libre de enfermedad de 40 meses ( $32 \%$ a 5 años), mientras

Tabla 4. Evaluación de anatomía patológica de las piezas resecadas

\begin{tabular}{|lrrr|}
\hline & & $\mathbf{n}$ & $\%$ \\
\hline Número de metástasis: & 1 & 33 & 50 \\
& 2 & 11 & 17 \\
& 3 & 7 & 11 \\
& 4 & 5 & 7 \\
& 5 o más & 10 & 15 \\
& Promedio: $2,7 \pm 2,9$ & & \\
& Rango: 1-16 & & 64 \\
Tamaño de metástasis $(\mathrm{cm}):$ & Menor de 5 & 42 & 26 \\
& 5-10 & 17 & 10 \\
& 10 o mayor & 7 & \\
& Promedio: $4,9 \pm 4,7$ & & 80 \\
Resultado de la cirugía: & Rango: 1-22 & & 9 \\
& R0 & 53 & 11 \\
\hline & R2 & 6 & 7 \\
\hline
\end{tabular}

R0: hígado libre de tumor, R1: borde microscópico positivo, R2: lesiones macroscópicas no resecadas.

ARTÍCULO DE 


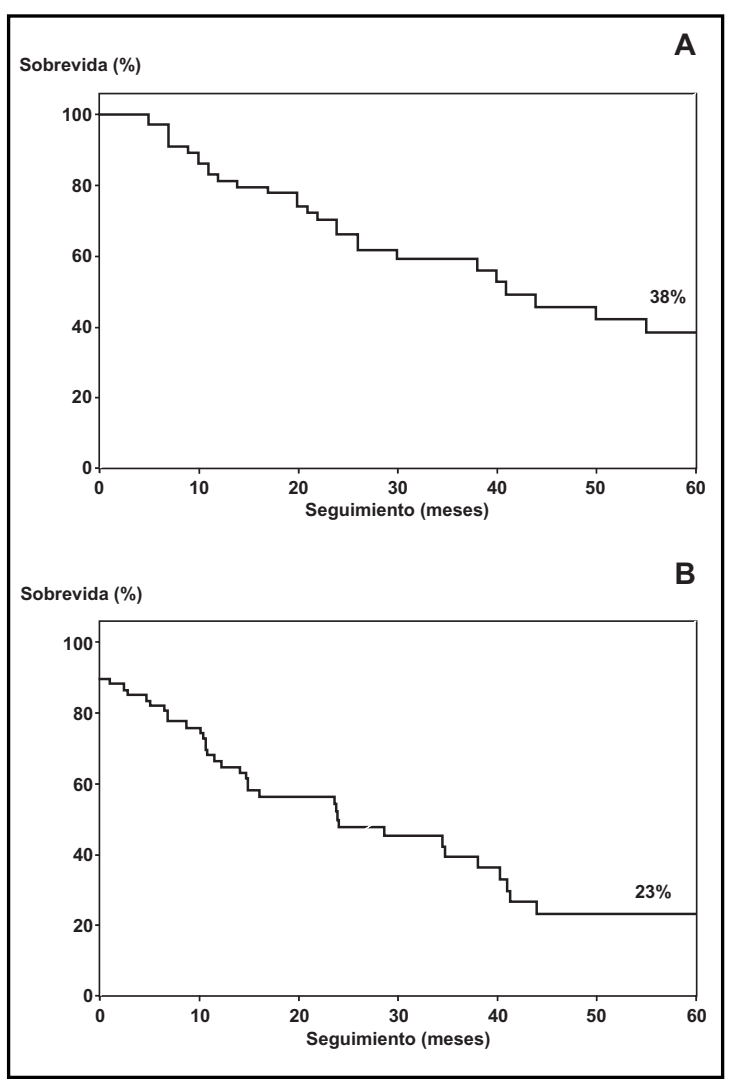

Figura 2. Sobrevida de pacientes con resección por MHCCR. Sobrevida global (A) y sobrevida libre de enfermedad hepática (B).

que con puntaje de 3 o más, ésta fue de 12 meses (9\% a 5 años) (Figura 4 ).

Durante los últimos 9 años del estudio se produjo un aumento significativo en las resecciones por año ( $p=0,003)$, así como también, del número de resecciones anatómicas $(p=0,04)$ y de la cantidad de segmentos resecados $(p=0,03)$ (Tabla 5). No se modificó la morbilidad y la mortalidad fue $0 \%$ en ambos períodos. Además, durante estos años se incrementó el número de pacientes con 3 o más FPMSK que fueron resecados ( $p=0,01$ ). También, se observó una mayor colaboración con el equipo de oncología médica, al indicarse quimioterapia adyuvante de segunda línea en $54 \%$ de los pacientes resecados (Tabla 5). Finalmente, en el segundo período del estudio se obtuvo un aumento significativo en la sobrevida: de 22 a 55 meses (de $21 \%$ a $45 \%$ a 5 años) (p =0,04) (Figura 5).

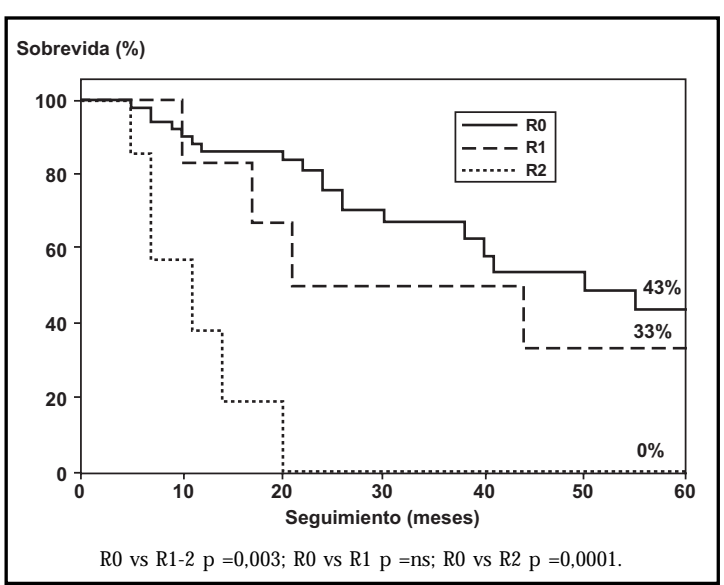

Figura 3. Sobrevida según resultado de cirugía realizada (R0-R1-R2).

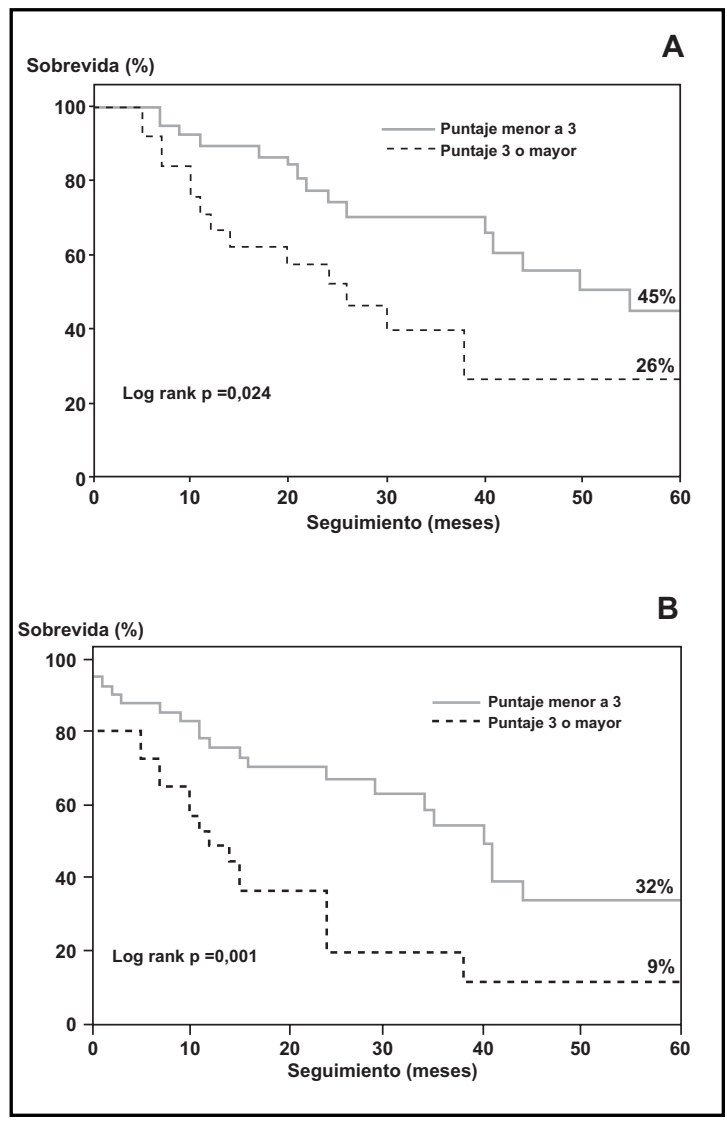

Figura 4. Sobrevida global (A) y sobrevida libre de enfermedad según puntaje de Memorial Sloan-Kettering Cancer Center (B). 
Tabla 5. Comparación de resecciones hepáticas por metástasis colorrectales en 2 períodos; 1990-1997 y 1998-2007

\begin{tabular}{|llll|}
\hline & $\mathbf{9 0 - 9 7}$ & $\mathbf{9 8 - 0 7}$ & $\mathbf{p}$ \\
\hline Pacientes & 14 & 52 & \\
Resecciones & 14 & 61 & \\
Resecciones por año & $1,8 \pm 1,5$ & $6,1 \pm 4,0$ & 0,01 \\
Sexo masculino & $57 \%$ & $72 \%$ & $\mathrm{~ns}$ \\
Edad (años) & $63 \pm 11$ & $60 \pm 12$ & $\mathrm{~ns}$ \\
Resecciones anatómicas & $50 \%$ & $77 \%$ & 0,04 \\
Segmentos resecados & $1,3 \pm 1,6$ & $2,5 \pm 1,8$ & 0,03 \\
Complicaciones & $35 \%$ & $34 \%$ & $\mathrm{~ns}$ \\
Mortalidad & $0 \%$ & $0 \%$ & $\mathrm{~ns}$ \\
Hospitalización (días) & $12 \pm 4$ & $10 \pm 6$ & $\mathrm{~ns}$ \\
Puntaje 3 o más MSK & $7 \%$ & $43 \%$ & 0,01 \\
Cirugía R0 & $71 \%$ & $84 \%$ & $\mathrm{~ns}$ \\
Quimioterapia 2a Línea & $0 \%$ & $54 \%$ & 0,0001 \\
\hline
\end{tabular}

MSK: Memorial Sloan-Kettering Cancer Center. (Datos en promedio \pm desviación estándar o porcentaje)

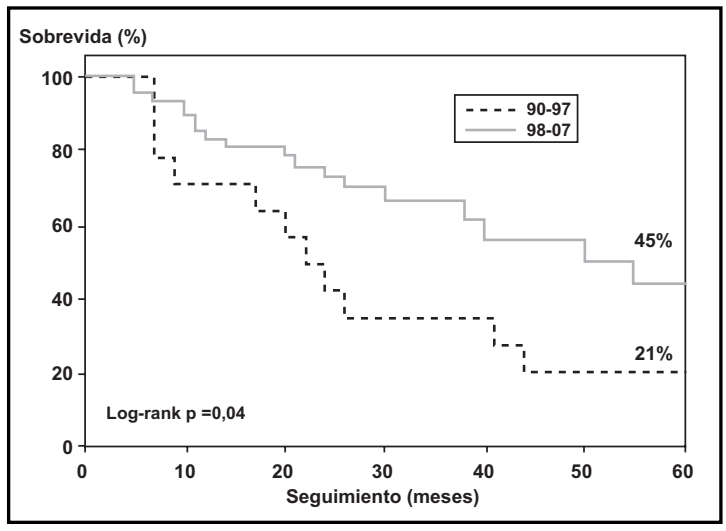

Figura 5. Sobrevida según período de la serie.

\section{DisCUSIÓN}

Estos resultados de sobrevida para un cáncer en etapa metastásica son significativamente mejor que la evolución natural de la enfermedad o que cualquier esquema de terapia descrito y son comparables a los publicados de centros extranjeros de referencia con sobrevida a 5 años de $28 \%$ a $45 \%{ }^{4-6,22}$

La morbilidad de $35 \%$ y mortalidad de 0, están en el rango de las mejores series extranjeras, con una morbilidad de alrededor de $30 \%$ y una mortalidad de $3 \% 6,22$. Esto, pese a la tendencia a realizar resecciones hepáticas mayores.

Existe controversia en el tipo de resecciones por MHCCR. Algunos autores proponen realizar resecciones locales o no anatómicas ${ }^{23}$ y otros, realizar resecciones hepáticas anatómicas ${ }^{24}$. Existen pocos trabajos que comparen ambas técnicas en forma adecuada ${ }^{25}$. Las resecciones anatómicas tendrían los beneficios de lograr un mejor margen y que pueden incluir metástasis en la pieza quirúrgica no pesquisadas previamente, con una morbilidad similar. El sangrado quirúrgico es más controlado, debido a que se siguen planos anatómicos en la transección hepática ${ }^{24}$. Las resecciones no anatómicas permiten preservar una mayor cantidad de parénquima hepático sano, lo que es de especial relevancia en pacientes con fibrosis 0 esteatosis hepática mayor. En esta serie realizamos $15 \%$ de resecciones extendidas, las que asociadas a resecciones locales o ablaciones por radiofrecuencia, permitió dejar libre de enfermedad a pacientes con MHCCR múltiples y bilaterales, preservando un adecuado volumen hepático residual.

También existe discusión sobre cuál es el mejor momento para resecar las metástasis sincrónicas con el primario colorrectal. Practicar la

ARtículo DE 
cirugía colónica y hepática diferidamente o resecar las metástasis en forma simultánea a la cirugía colorrectal. La cirugía en 2 tiempos es la opción que se ha preferido en muchos centros, aunque recientemente han aparecido grupos que proponen realizar la cirugía en forma simultánea ${ }^{26-29}$. Esta serie presenta $32 \%$ de resecciones simultáneas. Éstas se indicaron caso a caso, dependiendo de las condiciones generales del paciente, la magnitud y duración de la cirugía colorrectal y el tipo de cirugía hepática requerida, intentando no someter al enfermo a un trauma quirúrgico desmedido ni a un tiempo operatorio excesivo. En este trabajo, los pacientes seleccionados que fueron sometidos a una cirugía simultánea, no tuvieron una mayor morbilidad y ahorraron una nueva hospitalización, gastos y una nueva intervención que un porcentaje de pacientes rechaza en los plazos recomendados (3-6 meses). Dado que los números son pequeños, no podemos demostrar los beneficios de la cirugía simultánea y se deben esperar resultados de trabajos de mayor poder estadístico; sin embargo, representa una alternativa en pacientes seleccionados.

Es conocido que una proporción variable de los pacientes que se resecan por MHCCR presentan recurrencia en el remanente de hígado. En 30\% de los casos esta recidiva es exclusivamente hepática ${ }^{7}$. Por lo anterior, se han planteado las re-resecciones ante la aparición de nuevas metástasis 7,30 . En esta serie se realizaron 8 segundas hepatectomías, con buenos resultados y sobrevida a largo plazo. Es planteable realizar re-resecciones con resultados comparables a la primera hepatectomía ${ }^{31,32}$.

Se han descrito múltiples sistemas de puntaje para calcular el pronóstico de pacientes con MHCC sometidos a cirugía resectiva ${ }^{5,6,22,33}$ destacando el propuesto por los autores del Memorial Sloan Kettering Cancer Center que describe un sistema de 5 puntos $^{6}$. Encontramos una buena correlación pronóstica de éstos, con una significativa mejor sobrevida en los pacientes con puntajes

\section{REFERENCIAS}

1. Jarnagin W, Gonen M, Fong Y, Dematteo R, Ben-Porat L, LTTLE S ET AL Improvement in perioperative outcome after hepatic resection: analysis of 1,803 consecutive cases over the past decade. Ann Surg 2002; 236: 397-406. menores a 3. Este tipo de factores pronósticos, no deben ser usados para negarle la cirugía a un paciente en particular, sino que permiten seleccionar enfermos que deben ser sometidos a nuevas terapias adyuvantes. En esta serie tuvimos sobrevivientes en pacientes con puntaje elevado.

La quimioterapia en pacientes con MHCC ha probado ser efectiva como estrategia neoadyuvante $^{7}$. Existen dudas sobre cuál es el mejor esquema de quimioterapia ${ }^{34}$. Prácticamente todos los pacientes recibieron algún tipo de adyuvancia y más de la mitad de los enfermos tratados durante los últimos años recibieron un esquema de segunda línea. Creemos que el manejo óptimo de estos pacientes requiere trabajar en equipos multidisciplinarios.

Considerando el cambio de conducta hacia una cirugía resectiva más amplia con el objetivo de lograr una resección completa de las metástasis, destacando $15 \%$ de resecciones extendidas ${ }^{35}$, no observamos un aumento en la morbilidad ni mortalidad postoperatoria. Los pocos casos de insuficiencia hepática fueron autolimitados y los pacientes recuperaron su función hepática. Esta conducta se ha asociado a una mejoría en la sobrevida a 5 años, pese a que el grupo tenía más FPMSK. Si bien este resultado puede ser explicado por múltiples variables, pensamos que un elemento esencial, ha sido un enfrentamiento quirúrgico más agresivo con un aumento en el número de segmentos resecados.

Una de las limitaciones de este estudio es su carácter retrospectivo de una cohorte relativamente pequeña de pacientes, en relación a grandes centros.

Esta serie, confirma que la resección de MHC$\mathrm{CR}$ es una terapia segura que permite la curación de una enfermedad neoplásica avanzada. Estos resultados debieran ser considerados toda vez que se evalúa a un paciente con MHCCR y señala una estrategia para otro tipo de metástasis hepáticas de cánceres de origen diferente ${ }^{36,37}$.

2. Hughes K, Simons R, Songhorabodi S Adson M, Ilstrup D, FORTNER J ET AL. Resection of the liver for colorectal carcinoma metastases: a multi-institutional study of indications for resection. Surgery 1988; 103: 278-88.

3. Gayowsi TJ, Iwatsuki S, Madariaga JR, Selby R, Todo S, IRISH W ET AL. Experience in hepatic resection for metastatic colorectal cancer: analysis of clinical and 
pathological risk factors. Surgery 1994; 116: 703-11.

4. Scheele J, Stang R, Altendorf-Hofmann A, Paul M. Resection of colorectal liver metastases. World J Surg 1995; 19: 59-71.

5. Nordunger B, Guiguet M, Vailunt JC, Baluadur P, Boudjema K, Bache山ler P ET al. Surgical resection of colorectal carcinoma metastases to the liver. A prognostic scoring system to improve case selection, based on 1568 patients. Association Francaise de Chirurgie. Cancer 1996; 77: 1254-62.

6. Fong Y, Fortner J, Sun R, Brennan M, Blumgart L. Clinical score for predicting recurrence after hepatic resection for metastatic colorectal cancer analysis of 1001 consecutive cases. Ann Surg 1999; 230: 309-21.

7. Adam R, Delvart V, Pascal G, Valeanu A, Castaing D, Azoulay D ET AL. Rescue surgery for unresectable colorectal liver metastases downstaged by chemotherapy a model to predict long-term survival. Ann Surg 2004; 240: 644-58.

8. Penna C. Prise en charge des patients ayant un cancer du foie: Les métastases hépatiques des cancers colo-rectaux. Bull Cancer 2003; 90: 79-83.

9. Bengtsson G, Carlsson G, Hafstrom L, Jonson P. Natural history of patients with untreated liver metastases from colorectal cancer. Am J Surg 1981; 141: 586-9.

10. Butte JM, Jarufe N, Bellolo F, Zúñiga A, López $F$, Pinedo G ET AL. Factores pronósticos de pacientes con metástasis hepáticas de cáncer de colon y recto. Rev Chil Cir 2007; 59: 293-8.

11. Grothey A, Sargent D, Goldberg RM, Schmol HJ. Survival of patients with advanced colorectal cancer improves with the availability of fluorouracil-leucovorin, irinotecan, and oxaliplatin in the course of treatment. J Clin Oncol 2004; 22: 1209-14.

12. Saltz LB, Cox JV, Blanke C, Rosen LS, Fehrnbacher L, MOORE MJ ET AL. Irinotecan plus fluorouracil and leucovorin for metastatic colorectal cancer. N Engl J Med 2000; 343: 905-14.

13. Goldberg RM, Sargent DJ, Morton RF, Fuchs CS, RAMANATHAN RK, WILUAMSON SK ET AL. A randomized controlled trial of fluorouracil plus leucovorin, irinotecan, and oxaliplatin combinations in patients with previously untreated metastatic colorectal cancer. J Clin Oncol 2004; 22: 23-30.

14. Meyerhardt J, Mayer R. Systemic Therapy for Colorectal Cancer. N Engl J Med 2005; 352: 476-87.

15. Burmeister R, García C, Bannura G, Viliaman J, Benavides C, LeE K. Resección de metástasis hepáticas por cáncer colorrectal. Rev Chil Cir 2001; 53: 563-8.

16. FoNG Y. Surgical therapy of hepatic colorectal metastasis. CA Cancer J Clin 1999; 49: 231-55.

17. Bismuth H. Surgical Anatomy and Anatomical Surgery of the Liver. World J Surg 1982; 6: 3-9.

18. Terminology Committee of the IHPBA. The Brisbane 2000 terminology of hepatic anatomy and resectio- ns. HPB (Oxford) 2002; 4: 99-100.

19. Jarufe N, Martínez J, Guzmán S, Pacheco F, Guajardo M, IBÁÑEZ L. Técnica de resecciones hepáticas con disección suprahiliar del pedículo. Rev Chil Cir 2005; 57: 462-9.

20. Pringle J. Note on the arrest of hepatic hemorrhage due to trauma. Ann Surg 1908; 48: 501.

21. Clavien PA, Yadav S, Sindram D, Bentley RC. Protective effects of ischemic preconditioning for liver resection performed under inflow occlusion in humans. Ann Surg 2000; 232: 155-62.

22. Malik H, Prasad R, Halazun K, Aldoori A, Al-Mukhtar A, Gómez D et AL. Preoperative Prognostic Score for Predicting Survival After Hepatic Resection for Colorectal Liver Metastases. Ann Surg 2007; 246: 806-14.

23. Kokudo N, Tada K, Seki M, Otha H, Azekura K, Ueno $\mathrm{M}$ ET AL. Anatomical major resection versus nonanatomical limited resection for liver metastases from colorectal carcinoma. Am J Surg 2001; 181: 153-9.

24. YAsUi K, SHImisu Y. Surgical treatment for metastatic malignancies. Anatomical resection of liver metastasis: indications and outcome. Int J Clin Oncol 2005; 10: 86-96.

25. Dematteo R, Palese C, Jarnagin W, Sun R, Blumgart L, FoNG Y. Anatomic segmental hepatic resection is superior to wedge resection as an oncologic operation for colorectal liver metastases. J Gastrointest Surg 2000; 4: 178-84.

26. Martin R, Paty P, Fong Y, Grace A, Cohen A, Dematteo $\mathrm{R}$ ET AL. Simultaneous liver and colorectal resections are safe for synchronous colorectal liver metastasis. J Am Coll Surg 2003; 197: 233-41.

27. Tanaka K, Shimada H, Matsuo K, Nagano Y, Endo I, SEKIDO H ET AL. Outcome after simultaneous colorectal and hepatic resection for colorectal cancer with synchronous metastases. Surgery 2004; 136: 650-9.

28. Reddy S, Pawlik T, Zorzi D, Gleisner A, Ribero D, AssumpCAo L ET AL. Simultaneous resections of colorectal cancer and synchronous liver metastasis: a multi-institutional analysis. Ann Surg Oncol 2007; 14: 3481-91.

29. Thelen A, Jonas S, Benckert C, Spinelu A, LopezHanninen E, Rudolph B et AL. Simultaneous versus staged liver resection of synchronous liver metastases from colorectal cancer. Int J Colorectal Dis 2007; 22: 1269-76.

30. Adam R, Pascal G, Azoulay D, Tanaka K, Castaing D, BismuTH $H$. Liver resection for colorectal metastases: the third hepatectomy. Ann Surg 2003; 238: 871-83.

31. Petrowsky H, Gonen M, Jarnagin W, Lorenz M, Dematteo R, HeinRich S Et al. Second liver resections are safe and effective treatment for recurrent hepatic metastases from colorectal cancer. A bi-institucional analysis. Ann Surg 2002; 235: 863-71.

32. Thelen A, Jonas S, Benckert C, Schumacher G, LopezHanninen E, Rudolph B ET AL. Repeat liver resection

ARtíCULO DE 
for recurrent liver metastases from colorectal cancer. Eur J Surg Oncol 2007; 33: 324-8.

33. Zakaria S, Donohue J, Que F, Farnell M, Schleck C, ILSTRUP D ET AL. Hepatic Resection for Colorectal Metastases. Value for Risk Scoring Systems? Ann Surg 2007; 246: 183-91.

34. KeLuy C, CASSIDY J. Chemotherapy in metastatic colorectal cancer. Surgical Oncology 2007; 16: 65-70.

35. Guerra JF, Jarufe N, Martínez J, Guzmán S, Pacheco F, Guajardo M et al. Hepatectomía extendida. Una alternativa segura en lesiones hepatobiliares malignas. Rev Chil Cir 2007; 59: 430-5.

36. Martínez J, Jarufe N, González R, Alvarez M. Alternativas terapéuticas actuales de las metástasis hepáticas. Rev Méd Chile 2008; 136: 368-76.

37. Adam R, Chiche L, Aloia T, Elias D, Salmon R, Rivoire $M$ ET AL. Hepatic resection for noncolorectal nonendocrine liver metastases, analysis of 1452 patients and development of a prognostic model. Ann Surg 2006; 244: 524-35. 